\title{
APPLICATION OF THE SSCTRK NUMERICAL SIMULATION PROGRAM TO THE EVALUATION OF THE SSC MAGNET APERTURE*
}

\author{
T. Garavaglia, S. K. Kauffmann and R. Stiening \\ Superconducting Super Collider Laboratory ${ }^{\dagger}$ \\ 2550 Beckleymeade Ave. \\ Dallas, TX 75237
}

March 1990

\begin{abstract}
DISCLAIMER
This report was prepared as an account of work sponsored by an agency of the United States Government. Neither the United States Government nor any agency thereof, nor any of their employees, makes any warranty, express or implied, or assumes any legal liability or responsibility for the accuracy, completeness, or usefulness of any information, apparatus, product, or process disclosed, or represents that its use would not infringe privately owned rights. Reference herein to any specific commercial product, process, or service by trade name, trademark, manufacturer, or otherwise does not necessarily constitute or imply its endorsement, recommendation, or favoring by the United States Government or any agency thereof. The views and opinions of authors expressed herein do not necessarily state or reflect those of the United States Government or any agency thereof.
\end{abstract}

\footnotetext{
*Presented at the International Industrial Symposium on the Super Collider, Miami Beach, Florida, March 14-16, 1990.

†Operated by the Universities Research Association, Inc., for the U.S. Department of Energy under Contract No. DE-AC02-89ER40486.
} 


\title{
APPLICATION OF THE SSCTRK NUMERICAL SIMULATION PROGRAM TO THE EVALUATION OF THE SSC MAGNET APERTURE
}

\author{
T. Garavaglia. K. Kauffmann, and R. Stiening \\ Superconducting Super Collider Laboratory* \\ 2550 Beckleymeade Avenue \\ Dallas, Texas 75237
}

SUMMARY: The SSCTRK numerical simulation code has been used to estimate the benefit of increasing the SSC dipole aperture from 4 to $5 \mathrm{~cm}$. The increase in maximum amplitude of stable betatron oscillations depends on the level to which systematic errors have been corrected. Two cases have been studied, a highly corrected ring and a rirg with limited corrections. The maximum stable amplitude increase is approximately a factor of 1.3 in the case of the highly corrected ring and is approximately a factor of 1.6 in the case of the ring with limited systematic corrections. The aperture comparison has been made at 105 revolutions. Magnetic error assumptions are described in detail and a new table of errors suggested for future simulations is given.

\section{INTRODUCTION}

This report describes the application of the SSCTRK numerical simulation code developed by Ritson to the design of the Collider rings. Many principal features of the rings (magnet aperture. cell length, and injection energy) are determined by the requirement that the beam survive the long ( 40 minute) injection period without significant loss or emittance dilution. It is the injection process, that sets the design requirements, beciuse at injection, both the beam width and the relative magnetic field errors in the dipoles are largest. Numerical simulation is the only method presently available for establishing the long term behavior of a beam.

SSCTRK became available as a tool in the Summer of 1989. At that time, analytic estimates of tune shifts caused by persistent current multipoles indicated that the cell length should be reduced and the injection energy increased. For this reason, SSCTRK was used mainly to study the related issues of magnet aperture and the levels of tuning required to establish stable beams with suitable margins. All of the studies reported here have been made with the assumption of the shorter, $90-\mathrm{m}$ long, half-cell and the higher, $2 \mathrm{TeV}$, injection energy. A number of important considerations are not addressed by the SSCTRK simulation. The effects of time dependent persistent current multipoles, the requirement for careful control of superconductor magnetization, and the possible need for distributed correction of sextupole errors (if only for dipole alignment reasons) are examples of such considerations. Broadly speaking, the shorter cell length and higher injection energy removed these considerations from being first order concerns. SSCTRK has been used primarily to evaluate the benefit of increasing the magnet aperture from 4 to $5 \mathrm{~cm}$ and to establish the levels of tuning required to achieve the long term stability of betatron oscillations at a suitable amplitude.

At the time this work was carried oul $4 \mathrm{~cm}$ diameter dipoles had been produced in a variety of lengths. These dipoles were constructed in the course of studies undertaken to improve high field training and quench behavior. No production series had been made for the purpose of establishing distributions of random field errors nor had iteration of the magnet cross section to reduce allowed multipole errors been performed. For this reason the magnetic field errors used in the simulations r'ported here do not use input data des ived from measurements on SSC dipoles, but rely in:tead on the scaling of the magnetic measurements of dipoles produced in quantity for other accelerators.

\footnotetext{
"Operated by the Universities Research Association, Inc., for the U.S. Department of Energy under Contract No. DE-AC02-89ER 40486.
} 
For the purposes of numerical simulation, the magnetic field errors present in an ensemble of magnets are described in a simplified form. For each magnet, a multipole expansion of the magnetic field is made about a reference beam trajectory, in the SSC case, the geometrical center of the magnet coils. The particular form of the multipole expansion used in SSC literature is as follows:

$$
i B_{x}+B_{y}=B_{o} \Sigma\left(i A_{n}+B_{n}\right)(x+i y)^{n}
$$

In this expression $B_{o}$ is the central field of the dipole. The $B_{n}$ terms are called regular multipoles. Regular multipoles cause tune shifts. The $A_{n}$ terms are called skew multipoles. Skew multipoles couple horizontal and vertical modes of betatron oscillation.

In the simplified description used here, all information about the ensemble of magnets is condensed down to an average or "systematic" value for each multipole, $\left\langle A_{n}\right\rangle$ or $\left\langle B_{n}\right\rangle$ (the average is taken over all of the magnets in a ring), and a root mean square deviation $\sigma A_{n}$ or $\sigma B_{n}$ from the average. The rms deviation is frequently called a "random value" for a multipole. In making a numerical simulation, random numbers are chosen from Gaussian distributions having the appropriate average and rms values. The random numbers are appropriately assigned to each multipole in each magnet or block of magnets in the machine to be simulated. In order to avoid particularly fortunate or unfortunate combinations of errors from the random number generator, several machines are simulated, each from a different seed of the random number generator. In the work described here, the number of machines that have been simulated has been either eight or sixteen.

This description of an ensemble of magnets contains a potentially significant error because it has been assumed that the various multipole coefficients describing a magnet are uncorrelated. This assumption is incorrect. Future work should address this question.

If all magnets are measured, it is possible that ways of choosing the location of a magnet in the ring on the basis of its measurements may help to reduce the effects of random multipoles. In the work described here, magnet location has been chosen to reduce the effect of random regular sextupole $\sigma B 2$. The rules for making use of magnetic measurement data are not established. It is possible that before the installation of magnets in the SSC tunnel begins. progress will have been made on a magnet placement strategy.

\section{Random Multipoles}

The value of a multipole varies from magnet to magnet. There are a number of effects that contribute to the variation. These include variations in the persistent current magnetization of the superconducting cables (M. A. Green, SSC-N-377), variations in the permeability of the collar and the yoke, and variations in the placement of the current carrying cable. Such placement errors are frequently called "geometrical" errors. The SSC injection energy has been chosen high enough $(2 \mathrm{TeV})$ so that placement errors are dominant. If reasonable care is taken in the selection of materials the other errors should not be significant. Only placement errors have been considered in this report. Typical errors in the location of SSC magnet conductors are in the \pm 0.002 inch range.

In order to obtain expected multipole distributions for the $4 \mathrm{~cm} \mathrm{SSC} \mathrm{dipole} \mathrm{it} \mathrm{is} \mathrm{necessary}$ to scale results from the production measurements of other accelerator dipoles. A number of different scaling rules have been described in the Preliminary Report of the MagneticErrors Working Group of the SSC Aperture W'orkshop (SSC-7). The authors of SSC-7 used the average of three different methods to estimate errors. Their recommended set of $4 \mathrm{~cm}$ magnet errors (listed on page 66 of SSC-7) has been used as input for SSCTRK simulations with one exception. The estimated value for random sextupole has been reduced from 2.01 units to 0.4 units. This was done to maintain consistency with previous Central Design Group recommendations. The effect of random sextupole can be reduced by arranging magnets in the collider rings on the basis of measurements of the sextupole field error in each magnet. The reduced value of random sextupole simulates the effect of this magnet arrangement procedure. Several investigations have been made using SSCTRK which suggest that there may be little benefit from the reduction of random sextupole by magnet arrangement procedures. These investigations will be described later in this report. 
A somewhat simplified analysis suggests that bounds on the scaling law can be set by considering the following two plausible assumptions. The first is that the size of the construction errors is independent of the scale of the magnet. This assumption leads to the scaling law that the $n$th multipole varies as:

$$
\left(B_{n}^{\prime}, A_{n}^{\prime}\right)=\left(B_{n}, A_{n}, R_{s}^{-(n+1)}\right.
$$

In this expression $R_{S}$ is the rario of the old aperture to the new aperture. An equally plausibie assumption is that the construction errors are proportional to the size of the magnet. This second assumption leads to the scaling law that the $n$th multipole varies as

$$
\left(B_{n}^{\prime}, A_{n}^{\prime}\right)=\left(B_{n}, A_{n}\right) R_{s}^{-n}
$$

The multipole distributions for a $5 \mathrm{~cm}$ aperture dipole have been obtained by scaling the suggested values from SSC-7 using a scaling law that is intermediate between the two cases given previously.

$$
\left(B_{n}^{\prime}, A_{n}^{\prime}\right)=\left(B_{n}, A_{n}\right) R_{s_{s}^{-(n+1 / 2)}}
$$

Table 1. $\quad 4 \mathrm{~cm}$ and $5 \mathrm{~cm}$ dipole random multipole distributions used in SSCTRK simulations

\begin{tabular}{|l|ll|ll|l|}
\hline & $4 \mathrm{~cm}$ dipole & 5 cro dipole & Notes \\
\hline A1 & 0.7 & units & 0.49 & units & Probably too small \\
B1 & 0.7 & units & 0.49 & units & \\
\hline A2 & 0.62 & units & 0.35 & units & Obtained by sorting \\
B2 & 0.4 & units & 0.23 & units & Obtaned \\
\hline A3 & 0.69 & units & 0.32 & units & \\
B3 & 0.34 & units & 0.16 & units & \\
\hline A4 & 0.14 & units & 0.051 & units & \\
B4 & 0.59 & units & 0.22 & units & \\
\hline A5 & 0.16 & units & 0.047 & units & \\
B5 & 0.059 & units & 0.017 & units & \\
\hline A6 & 0.034 & units & 0.008 & units & \\
B6 & 0.075 & units & 0.018 & units & \\
\hline
\end{tabular}

The values of all multipoles with $n>6$ were taken to be zero to speed the computation.

It is useful to compare these input data with actual magnetic measurements of production magnets. The data for the Tevatron have been published $(\mathrm{H}$. Edwards. Proceedings of the 12th International Conference on High Energy Accelerators, page 7). Recent HERA data on the first 156 dipoles measured have also been made available (Peter von Handel. private communication). For the purpose of scaling to the SSC $4 \mathrm{~cm}$ aperture, the same $n+1 / 2$ scaling law used previously has been adopted:

$$
\left(B_{n}^{\prime}, A_{41}^{\prime}\right)=\left(B_{n}, A_{n}\right) R_{\bar{s}}^{-(n+1 / 2)}
$$


Table 2. HERA and Tevatron Magnetic Measurements Scaled to the SSC $4 \mathrm{~cm}$ Aperture (Random Multipoles)

\begin{tabular}{|c|c|c|c|}
\hline Multipole & HERA & Tevatron & SSCTRK Simulation \\
\hline A1 & 1.74 & 0.80 & 0.70 \\
B1 & 0.68 & 0.57 & 0.70 \\
\hline A2 & 0.35 & 0.96 & 0.62 \\
B2 & 2.00 & 2.80 & 2.01 Sorted to 0.4 \\
\hline A3 & 0.52 & 0.92 & 0.69 \\
B3 & 0.16 & 0.49 & 0.34 \\
\hline A4 & 0.11 & 0.23 & 0.14 \\
B4 & 0.36 & 0.58 & 0.59 \\
\hline A5 & 0.07 & not applicable & 0.16 \\
B5 & 0.04 & not applicable & 0.06 \\
\hline A6 & 0.03 & not applicable & 0.034 \\
B6 & 0.07 & 0.22 & 0.075 \\
\hline
\end{tabular}

Note: The Tevatron measurements were made at 660 amps (injection) whereas the HERA measurements were made at $5000 \mathrm{amps}$ (high energy).

The above table shows that the random skew quadrupole ( $\sigma A 1)$ in the HERA dipole is much larger than the value used in the SSCTRK simulation. Skew quadrupole comes from an up-down asymmetry in the dipole. It is usually associated with differences in the dimensions of the upper coils and the lower coils. In the Tevatron dipoie the iron yoke was not adjacent to the collared coil. The coil position could be adjusted to null the quadrupole components. The SSC dipole and the HERA dipole do not have the provision for such an adjustment. The manufacturers of the HERA dipoles attempted to reduce the random skew quadrupole by measurement and re-shimming of the coils. The value given in the table above can not easily be reduced. (Peter Schmuser, private communication) In future simulations the value of $\sigma \mathrm{Al}$ should be increased from 0.7 units to 1.75 units.

\section{Systematic Multipoles}

The average or systematic value of a multipole may be non-zero for a variety of reasons. All multipoles will have non-zero averages because a collider ring contains a finite number of magnets.

The .ms width of this average is:

$$
\sigma<A_{n}, B_{n}>=\frac{\sigma\left(A_{n_{1}} B_{n}\right)}{\sqrt{n}}
$$

In this expression $n$ is the number of magnets in a collider ring. The collider rings contain 4800 magnets each. Random multipole sigmas are typically 0.5 units. The statistical value of systematic multipoles will be typically less than 0.01 units. These statistical systematics do not have a significant influence on the aperture.

Multipoles allowed by dipole symmetry, B2, B4, B6. etc. may have large values either caused by superconductor magnetization or by errors in the cross section. Superconductor magnetization multipoles have been calculated by Green for the C358 cross section (Progress on Niagnetization Calculations for the SSC Magnets. M. A. Green, presentation to the MSIM, 17 January 1990). The values for 1 and $2-\mathrm{TeV}$ field levels of the $4 \mathrm{~cm}$ SSC dipole are given in Table 3. The superconducting filament diameter used to obtain these results is 6 microns.

Table 3. $4 \mathrm{~cm}$ SSC Dipole Persistent Current Systematic Multipoles

\begin{tabular}{|c|r|r|}
\hline Multipole & \multicolumn{1}{|c|}{$1 \mathrm{TeV}$} & $2 \mathrm{TeV}$ \\
\hline $\mathrm{B} 0$ & -15.06 units & -6.00 units \\
\hline $\mathrm{B} 2$ & -8.44 units & -3.29 units \\
\hline $\mathrm{B} 4$ & 0.69 units & $(0.23$ units \\
\hline $\mathrm{B} 6$ & -0.16 units & -0.06 units \\
\hline $\mathrm{B} 8$ & 0.06 units & 0.02 units \\
\hline
\end{tabular}


The scaling of persistent current multipoles to other magnet apertures depends on the way the magnet cross section is changed. If all components. including the dimensions of the superconducting coils are scaled together. persistent current multipoles scale as $R_{s}^{-n}$. It is assumed that the dimensions of the superconducting filament have not been changed when the magnet size is changed.

Transport current in the superconducting cable will generate allowed multipoles, the dipole for example, in a manner which depends on the location of the conductor. The magnet designer will attempt to inake the $\mathrm{B} 0$. or dipole multipole, as large as possible while suppressing other allowed multipoles.

The SSC 358 dipole coil assembly contains many copper wedges. For this reason there aie enough dimensions that can be adjusted so that all systematic allowed multipoles below $\mathrm{B} 14$ can be made to have, in principle at least, very small values (G. Morgan, CDG-N. 342). The Tevatron magnet, in contrast, does not have wedges. The only adjustable dimensions in the Tevatron magnet are the pole angles of the inner and outer coils. These angles are chosen to null the sextupole and decapole. The Tevatron has large values of higher muitipoles (see Table 5).

The cross section of a dipole coil as manufactured will differ from expectations based on the design. This is because actual dimensions differ from design dimensions. For example, at the present time the average value of B4 (other than that due to persistent current) in the $4 \mathrm{~cm}$ SSC dipole is 0.6 units. G. Morgan has given a prescription for reducing this value $(\mathrm{G}$. Morgan, SSC-MD-241). The ends of the magnet coil may also contribute $t()$ allowed multipoles. It is reasonable to assume that the coil geometry contribution to the values of allowed multipoles, including the effects of ends, can be reduced to a value small in comparison with persistent current value.

Unallowed systematic multipoles occur when the magnet symmetry is broken. Symmetry is broken, for example, by buswork, coil ends, coil sagitta, and gravity. For example, a small length of unpaired conductor located at a distance of $R_{B} \mathrm{~cm}$ from the center of the dipole produces a systematic multipole distribution as follows:

$$
\left(B_{n}, A_{n}\right)=\left(\frac{l_{B}}{l_{M}}\right)\left(\frac{2}{R_{B} \text { (meters) }}\right)\left(\frac{R_{\text {ref }}}{R_{B}}\right)^{n}
$$

In this expression. $l_{B}$ is the length of unpaired buswork, $l_{M}$ is the length of the magnet, and $R_{\text {ref }}$ is the reference radius for magnetic measurements. The transfer function (1 Tesla/kA) for the SSC $4 \mathrm{~cm}$ dipole has been assumed. As an example, Table 4 shows the effect on a 15 -meter-long magnet of $5 \mathrm{~cm}$ of unpaired conductor located at a distance of $2.5 \mathrm{~cm}$ from the axis.

Table 4. Systematic multipoles in a 15 -meter-long magnet caused by $5 \mathrm{~cm}$ of unpaired conductor located $2.5 \mathrm{~cm}$ from the dipole axis

\begin{tabular}{|l|l|}
\hline B1. A1 & 0.107 units \\
\hline B2. A2 & $0 .(143$ units \\
\hline B3. A3 & 0.017 units \\
\hline B4. A4 & 0.007 units \\
\hline
\end{tabular}

The systematic multipoles which were used for the input to numerical simulations described in this report were chosen to be the persistent current value, if allowed, and 0.05 units, if not allowed. At the time when these villues were selected, an attempt was being made to reduce the systematic decapole calused by a magnet cross section error. The value of 0.05 units was considered to be a difficult to achieve goal at that time. The signs of the unallowed systematics were all chosen to be the same to be consistent with the model of unpaired conductor. This assumption is not as important as it seems because even order systematics act only through momentum feed down. The tune shifts caused by momentum teed-down terms change sign every half synchrotron period.

Table 5 gives a comparison between the SSCTRK simulation assumptions and the measured data from HERA and TCväún niagnets. The HERA data have been taken at a high field level where persistent current multipoles are small. 
Table 5. HERA and Tevatron Magnetic Measurements Scaled to the SSC $4 \mathrm{~cm}$ Aperture (Systematic Multipoles)

\begin{tabular}{|c|c|c|c|}
\hline Multipole & HERA & Tevatron & SSCTRK Simulation \\
\hline$\langle\mathrm{A} 1\rangle$ & -0.293 & -0.007 & 0.00 (iuned to 0$)$ \\
$\langle\mathrm{B} 1\rangle$ & -0.038 & 0.044 & 0.00 (tuned to 0 ) \\
\hline$\langle\mathrm{A} 2\rangle$ & -0.164 & 0.060 & 0.05 \\
$\langle\mathrm{~B} 2\rangle$ & 0.175 & -2.567 & -3.00 (tuned to 0$)$ \\
\hline$\langle\mathrm{A} 3\rangle$ & 0.093 & -0.016 & 0.05 \\
$\langle\mathrm{~B} 3\rangle$ & 0.068 & -0.093 & 0.05 \\
\hline$\langle\mathrm{A} 4\rangle$ & 0.029 & -0.012 & 0.05 \\
$\langle\mathrm{~B} 4\rangle$ & 0.274 & 0.036 & 0.05 (tuned to .2$)$ \\
\hline$\langle\mathrm{A} 5\rangle$ & -0.017 & not applicable & 0.05 \\
$\langle\mathrm{~B} 5\rangle$ & -0.014 & not applicable & 0.05 \\
\hline$\langle\mathrm{A} 6\rangle$ & -0.002 & not applicable & 0.05 \\
$\langle\mathrm{~B} 6\rangle$ & 0.047 & 0.887 & -0.07 \\
\hline
\end{tabular}

Note: The Tevatron measurements were made at 660 amps (injection) whereas the HERA measurements were made at 5000 amps (high energy).

The data of Table 5 suggest that the SSCTRK simulation values of high order unallowed multipoles may have been overestimated and that the values of low order multipoles may have been underestimated. The calculation given previously of the multipole errors caused by unpaired buswork support this conclusion.

\section{SUGGESTED MULTIPOLES FOR FUTURE SIMULATIONS}

A revised set of multipole expectations for 4 and $5 \mathrm{~cm}$ magnets of the SSC design is given in Table 6 . The values for the $4 \mathrm{~cm}$ magnet were chosen in the following way:

1. Random multipole $\mathrm{rms}$ widths are those taken from SSC-7, page 66 except for the skew quadrupole (Al) which has been increased by a factor of 2.5 on account of a now different method of magnet construction.

2. The values in Table 6 do not assume any kind of magnet sorting. Future simulations should employ sorting only if it is established that it is beneficial.

3. Systematic allowed multipoles are equal to persistent current values. It has been assumed that iteration of the magnet cross section will reduce geometrical errors to levels that are considerably smaller than the persistent current values.

4. Systematic unallowed multipoles have been estimated by using Tevatron and HERA data in combination with the unpaired conductor model. $A$ value for the systematic octupole of 0.06 units has been chosen. Other unallowed multipoles are obtained from the octupole value by assuming that the error occurs at a radius of $2.5 \mathrm{~cm}$ and scales according to the unpaired conductor model.

5. Table 6 does not take into account possible correction schemes. For example. the systematic regular sextupole $\langle$ B2 $\rangle$ obviously needs to be highly corrected.

Values for a $5 \mathrm{~cm}$ aperture dipole are obtained by scaling the $4 \mathrm{~cm}$ values in the following way:

1. Random multipoles scale as $(4 / 5)^{n+1 / 2}$.

2. Persistent current systematic multipoles scale as $(4 / 5)^{n}$.

3. Ünailowed systematic mulupoles scale as $(4 / 5)^{n}$. 
Table 6. Suggested Multipoles for Future Simulation of $2 \mathrm{TeV}$ Injection

\begin{tabular}{|c|c|c|}
\hline & $4 \mathrm{~cm}$ & $5 \mathrm{~cm}$ \\
\hline$\langle\mathrm{A} 1\rangle$ & 0.37 & 0.30 \\
$\sigma \mathrm{A} 1$ & 1.75 & 1.25 \\
\hline$\langle\mathrm{B} 1\rangle$ & 0.37 & 0.30 \\
$\sigma \mathrm{B} 1$ & 0.70 & 0.50 \\
\hline$\langle\mathrm{A} 2\rangle$ & 0.15 & 0.10 \\
$\sigma \mathrm{A} 2$ & 0.62 & 0.35 \\
\hline$\langle\mathrm{B} 2\rangle$ & -3.29 & -2.11 \\
$\sigma \mathrm{B} 2$ & 2.01 & 1.15 \\
\hline$\langle\mathrm{A} 3\rangle$ & 0.06 & 0.031 \\
$\sigma \mathrm{A} 3$ & 0.69 & 0.32 \\
\hline$\langle\mathrm{B} 3\rangle$ & 0.06 & 0.031 \\
$\sigma \mathrm{B} 3$ & 0.34 & 0.16 \\
\hline$\langle\mathrm{A} 4\rangle$ & 0.024 & 0.01 \\
$\sigma \mathrm{A} 4$ & 0.14 & 0.05 \\
\hline$<\mathrm{B} 4\rangle$ & 0.23 & 0.09 \\
$\sigma \mathrm{B} 4$ & 0.59 & 0.22 \\
\hline$\langle\mathrm{A} 5\rangle$ & 0.01 & 0.004 \\
$\sigma \mathrm{A} 5$ & 0.16 & 0.047 \\
\hline$\langle\mathrm{B} 5\rangle$ & 0.01 & 0.004 \\
$\sigma \mathrm{B} 5$ & 0.059 & 0.017 \\
\hline$\langle\mathrm{A} 6\rangle$ & 0.004 & 0.0012 \\
$\sigma \mathrm{A} 6$ & 0.034 & 0.008 \\
\hline$\langle\mathrm{B} 6\rangle$ & -0.060 & -0.016 \\
$\sigma \mathrm{B} 6$ & 0.075 & 0.018 \\
\hline & & \\
\hline
\end{tabular}

\section{MAGNET FIELD ERRORS (QUADRUPOLE MAGNETS)}

The SSCTRK simulation results given in this report describe a collider ring constructed with error-tree quadrupoles. Although the effects of quadrupole errors are estimated to be small, future simulation should include such errors. There is one systematic error that should be considered, $\langle$ B5 $>$ caused by persistent currents. There are two random errors that should be considered. a quadrupole-to-quadrupole variation in length, and a random roll angle.

The persistent current $<$ B $5>$ has been estimated by M. A. Green (private communication) for an early version of the SSC $4 \mathrm{~cm}$ quadrupole. The SSCTRK code uses as input two parameters QB5F and QB5D, one for each of the two families of quadrupoles. These parameters are equal and of opposite sign since the same quadrupoles are used for both families. QB5F and QB5D are defined as follows:

$$
\mathrm{QB5X}=10^{4}\left(\frac{L q}{L b}\right) \frac{\mathrm{B}_{5}(1 \mathrm{~cm})}{\left.\mathrm{B}_{0} \text { (Dipole }\right)},
$$

where $L q$ is the length of the quad and $L b$ is the total length of the bending magnets in a half cell. $B 5(1 \mathrm{~cm})$ is the magnetic field due to persistent currents in the quadrupole at a radius of $1 \mathrm{~cm}$. Green estimates that $B 5(1 \mathrm{~cm}) / B 1(1 \mathrm{~cm})=1.36 \times 10^{-4}$. This leads to the following values:

$$
\begin{aligned}
\text { QB5F } & =-1) .025 \\
\text { QB5D } & =+(0.025
\end{aligned}
$$

A rough estimate on the rms value of the quadrupole-to-quadrupole variation in gradient length product is $(0.001$. The rms value of the random roll angle is 0.0005 radians.

At the present time a prototype quadrupole is being constructed. Better estimates of the quadrupole field errors can be expected when the prototypes are measured. 


\section{NUMERICAL SIMULATION-OTHER INPUT CONDITIONS}

\section{Operating Tunes}

The tune values chosen for all of the simulation results presented here are $Q x=X X X .425$, $Q y=X X X .410$. These values were chosen on the basis of Main Ring and Tevatron experience. This operating point is clear of all resonances below 9 th order (see Figure 1).

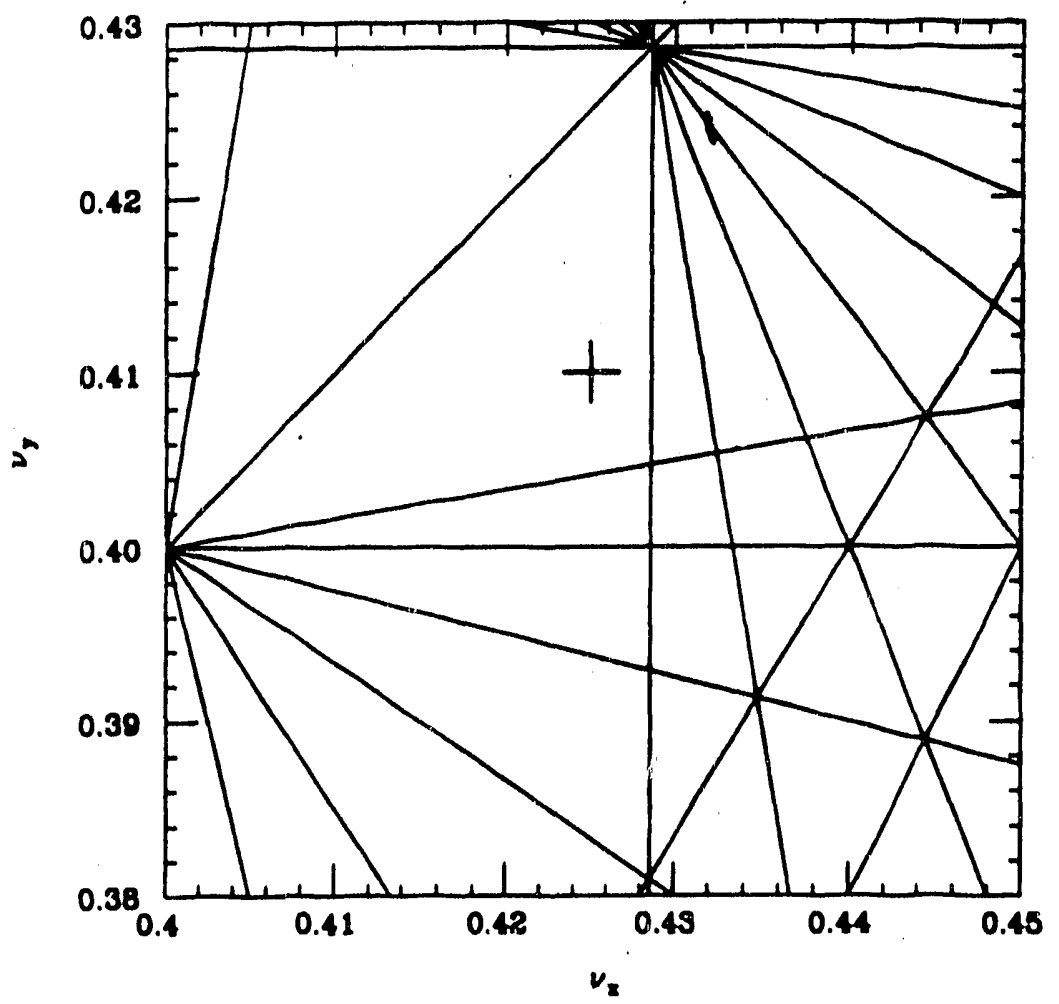

Figure 1. The cross marks the operating point chosen for SSCTRK simulation. All resonance lines below 9th order are shown.

\section{Lattice Parameters}

The half cell length is 90 meters and the betatron phase advance/cell is 95 degrees in all the simulations described in this report. The phase advance/cell differs from the Central Design Group recommendation of 90 degrees because it was thought that the ingt be a resonance between the beam and the lattice at 90 degrees/cell. Thus far simulations made to investigate this possibility have not shown a significant effect.

\section{Alignment Errors}

All magnetic elements have been assumed to be aligned with respect to the zero momentum error closed orbit of the beam with an rms deviation of $1.0 \mathrm{~mm}$.

\section{Radio Frequency Parameters and Momentum Spread}

The RF frequency and voltage have been chosen to be $375 \mathrm{MHz}$ and $20 \mathrm{MV}$. The maximum fractional momentum error of the tracked protons has been set at $5 \times 10^{-4}$. The corresponding value for the maximum longitudinal position error of the protons is $16.4 \mathrm{~cm}$.

The momentum error is much larger than the minimum that might in principle be present in a low current bunch injected into the collider rings. Past experience with proton rings suggests that longitudinal stability of high current beams is enhanced by increasing the bunch length. Since the bunch length that accompanies a $5 \times 10^{-4}$ fractional momentum error even at the injection energy is rnuch smaller than the low beta value at the interaction point and since the beam size due to dispersion, $\eta(\delta P / P)$, is only $1 \mathrm{~mm}$ this choice of momentum eror is reasonable. 


\section{Power Supply Ripple}

These simulations are made with the assumption that power supply (or tune) ripple has been reduced to the point where it does not affect the results. Therefore, no power supply ripple has been used in these simulations.

\section{RESULTS}

The beam will need to circulate without loss for approximately 40 minutes or 10 revolutions. Ideally all tracking should be done for this number of revolutions. Tracking for $10^{7}$ revolutions takes approximately 100 hours of CPU time on the Cray 2 or the NEC SX2, the two computers which were used for this work. One hour runs, $10^{5}$ revolutions, have been used to quickly evaluate various error configurations, and longer runs have been made for interesting cases.

The injection conditions at the start of each simulation are as follows. Particles are injected such that their betatron amplitude in the two transverse directions is equal when measured at the maximum value of the appropriate beta function. Betatron amplitude is measured with respect to the closed orbit. All particles are injected with a momentum error of $(\delta P) / P=5 \times 10^{-4}$.

In a typical run. 16 different machines are simulated simultaneously. (The machines differ only in the seed of the random number generator which is used to select the multipole coefficients for each magnet.) For each machine, particles are started with four different betatron amplitudes, typically $10,10.33,10.66$, and $11 \mathrm{~mm}$. Particles are circulated until they are lost. In these simulations a particle is considered to be lost if its distance from the closed orbit exceeds $21 \mathrm{~mm}$. If a particle is lost, another particle is launched at the location of the loss at an initial amplitude which is typically $1 \mathrm{~mm}$ smaller than the initial amplitude of the particle that was lost. The results are given as a plot which shows the number of revolutions before loss as a function of initial betatron amplitude. For the purpose of estimating safety margins in the SSC design it should be noted that the rms betatron amplitude for the 2-TeV specification SSC emittance is $0.375 \mathrm{~mm}$.

The SSCTRK code does not incorporate interaction regions. Interaction region beta values at injection are modest. It is not expected that this omission will significantly alter the results presented here.

All large accelerators employ magnetic correction systems to compensate the effects of errors. Two cases will be described in this report. The cases differ in the extent to which compensation is used to enlarge the aperture. The first case illustrates a highly corrected accelerator. In this casse all systematic field errors have been corrected. The second case illustrates a moderately corrected accelerator. This case incorporates corrections similar (in concept, though not in detail) to those of the Fermilab Main Ring. These corrections are typical of past accelerator practice.

Details of diagnostic and correction systems are not contained within the SSCTRK simulation with one exception. the natural chromaticity sextupoles. The natural chromaticity of the quadrupole lattice is compens'ted by sextupoles located adjacent to the quadrupoles. All other errors are corrected essentially at the point of origin. This is accomplished in two different ways. The first way of correcting an error is simply to enter a reduced or zero value in the SSCTRK input data set. The second way is accomplished within the SSCTRK program. SSCTRK evaluates the strength of a multipole by making an appropriate computation using the multipole error in each magnet. The correction is made by subaracting a computed vilue from the value of each magnet.

\section{The Highly' Corrected Machine}

Table 7 shows the corrections that have been made in this calse.

Figure 2 shows the simulation of $10^{5}$ turns of the highly corrected machine made with $4 \mathrm{~cm}$ diameter bore dipoles. In this report the left edge of the distribution of points is called the aperture. Figure 2 shows that the aperture for a few revolutions is $9 \mathrm{~mm}$ and that the aperture for 105 revolutions is $6 \mathrm{~mm}$. Since the rms transverse size of an SSC spec emittance beam is $\left(0.375 \mathrm{~mm}\right.$. this machine has ample sperture for $10^{5}$ sevolutions. This machine has not been simulated beyond $100^{5}$ revolutions. 
Table 7. The Highly Corrected Machine

\begin{tabular}{|c|c|}
\hline $\begin{array}{l}<A 1>: \\
<A 2> \\
<A 3> \\
<A 4>: \\
<A 5>: \\
<A 6>\end{array}$ & $\begin{array}{l}\text { Statistical value (approximately } 0.005 \text { units) } \\
\text { Statistical value } \\
\text { Statistical value } \\
\text { Statistical value } \\
\text { Statistical value }\end{array}$ \\
\hline $\begin{array}{l}<\mathrm{B} 1> \\
<\mathrm{B} 2> \\
<\mathrm{B} 3> \\
<\mathrm{B} 4> \\
<\mathrm{B} 5> \\
<\mathrm{B} 6>\end{array}$ & $\begin{array}{l}0 \\
0 \\
\text { Statistical value } \\
\text { Statistical value } \\
\text { Statistical value } \\
\text { Statistical value }\end{array}$ \\
\hline $\begin{array}{l}\text { Chromaticity: } \\
\text { Quadrupole errors: }\end{array}$ & $\begin{array}{l}0 \\
\text { none }\end{array}$ \\
\hline
\end{tabular}

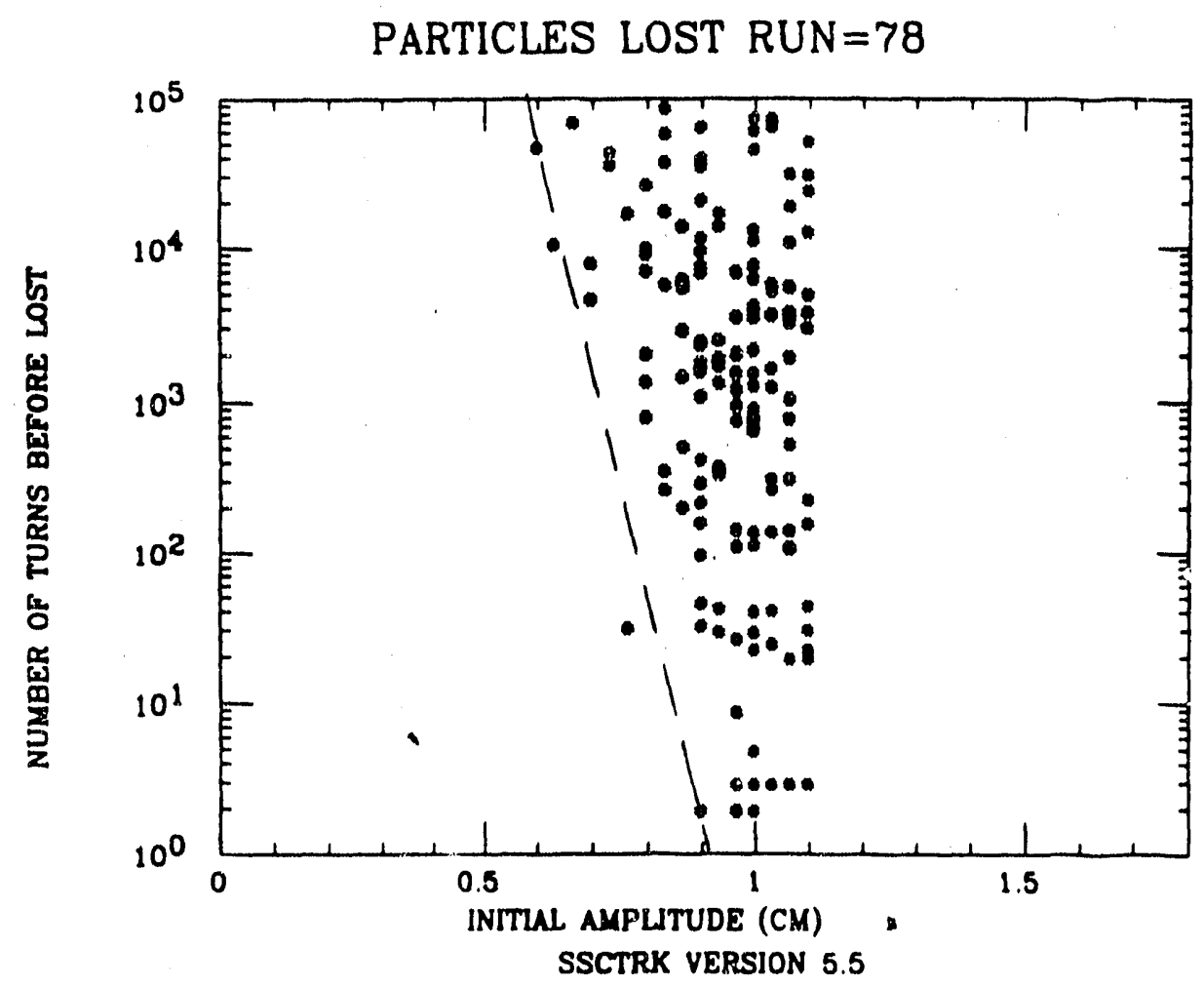

Figure 2. Numerical simulation of the collider ring with $4 \mathrm{~cm}$ dipole errors. This simulation is of the highly corrected ring. The dotted line is drawn for comparison with Figure 4 , the $5 \mathrm{~cm}$ dipole error highly conrected ring.

We have made a simulation identical to that of Figure 2 in all respects except for the value of random sextupole $\sigma \mathrm{B} 2$. Figure 3 shows the effect of increasing the value of the randorn sextupole a factor of five from 0.4 to 2.01 units. There is not a significant difference between the results of Figure 2 and those of Figure 3.

When the aperture of the dipole is increased from 4 to $5 \mathrm{~cm}$, the rms values of random multipoles decrease according to the $n+1 / 2$ law discussed earlier. It is plausible that the amplitude at which betatron oscillations are stable would increase to the point where the field errors in the $5 \mathrm{~cm}$ magnet are identical to the field errors in the $4 \mathrm{~cm}$ magnet. This occurs according to the following ratio for the $n$th multipole:

$$
R_{5}=R_{4}\left(\frac{5}{4}\right) !+(1 / 2 n)
$$




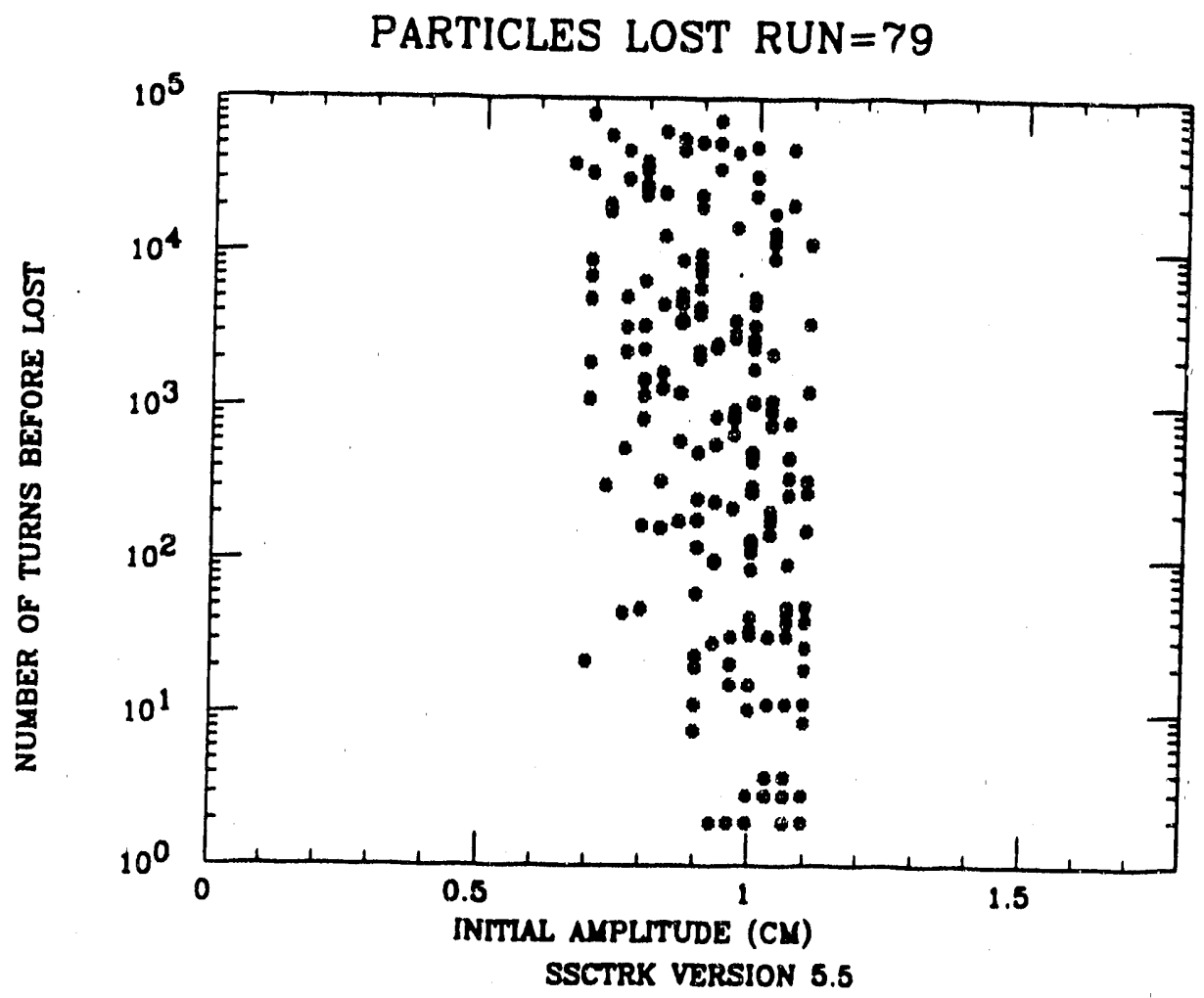

Figure 3. Numerical simulation of the collider ring with $4 \mathrm{~cm}$ dipole errors with the magnets not sorted on the basis of the value of the regular sextupole (B2). This simulation is of the highly corrected ring. It should be compared with Figure 2 where random value of regular sextupole has been reduced by a factor of 5 through sorting.

In this expression $R_{4}$ is some radius in the $4 \mathrm{~cm}$ magnet. $R_{5}$ is the radius in the $5 \mathrm{~cm}$ magnet at which the field error due to the $n$th multipole is equal to that in the $4 \mathrm{~cm}$ magnet at a radius $R_{4}$. If the betatron aperture is dominated by very high order multipoles the increase in aperture is $5 / 4$. Since the aperture appears not to be influenced by random sextupole, the octupole $(n=3)$ can be taken as the lowest multipole contributing to the aperture. The aperture should then scale in the ratio of 1.3 if it is octupole dominated or made with $5 \mathrm{~cm}$ manery high multipoles. This conclusion is supported by a simulation at 100,000 revers parameters. Figure 4 shows this result. The aperture is $8.5 \mathrm{~mm}$ at 100,000 revolutions. The dotted lines in Figures 2 and 4 are drawn according to a
1.28 radius ratio.

\section{The Plausibly Corrected Machine}

Table 8 shows the corrections that have been made in this case. These corrections are called plausible because it is likely that they could be made early in the commissioning of
the machine.

The plausibly tuned machine differs from the highly corrected machine only in the values chosen for systematic multipoles. Figure 5 shows the results of $10^{5}$ revolution tracking of the plausibly tuned machine. The presence of small, uncorrected. systematic multipoles reduces the aperture for stable betatron motion considerably. A longer term tracking (13 minutes of real time) of the plausibly tuned machine is shown in Figure 6. This tracking has been made on eight rather than sixteen machines in order to speed the
computation.

The effect of reducing the random sextupole by magnet placement has been investigated by increasing the value of random sextupole from 0.4 units to 2.01 units. Figure 7 shows a simulation made with $\sigma \mathrm{B} 2=2.01$ units. It should he compared with Figure 5 . Thic is practically no effert on the stable betatron aperture. 


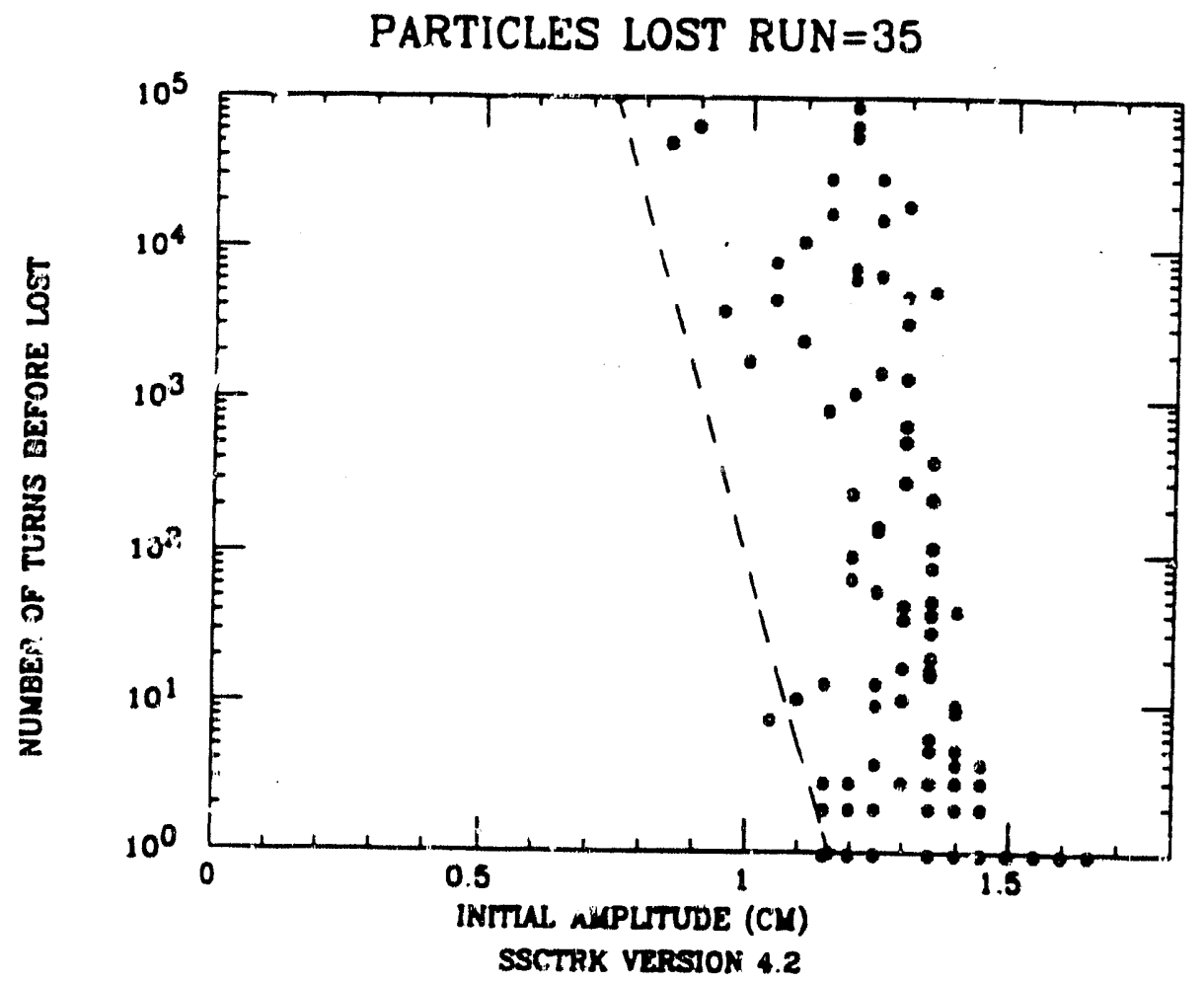

Figure 4. Numerical simulation of the collider ring with $5 \mathrm{~cm}$ dipole errurs. This simulation is of the highly corrected ring. The dotted line is srawn at an amplitude 1.28 times larger than the ciotted line in Figure 2 to illustrat: the scaling of stable betatron aperture.

Table 8. The Plausibly Corrected Machine

\begin{tabular}{|c|c|}
\hline $\begin{array}{l}<A 1>: \\
<A 2>: \\
<A 3> \\
<A 4> \\
<A 5> \\
<A 6 \%:\end{array}$ & $\begin{array}{l}0 \\
0.05+\text { Statistical value } \\
0.05+\text { Statistical value } \\
0.05+\text { Statistical value } \\
0.05+\text { Statistical value } \\
0.05+\text { Statistical value }\end{array}$ \\
\hline $\begin{array}{l}<B 1> \\
<B 2> \\
<B 3> \\
<B 4> \\
<B 5> \\
<B 6>\end{array}$ & $\begin{array}{l}0 \\
\text { See chromaticity errú ceiow } \\
0.05+\text { Statistical vaiiue } \\
0.05+\text { Statistical value } \\
0.05+\text { Statistical value } \\
-0.07+\text { Statistical value }\end{array}$ \\
\hline $\begin{array}{l}X \text { chromaticity: } \\
\text { Y chromaticity: } \\
\text { Quaddrupole error }\end{array}$ & $\begin{array}{l}+5 \text { units } \\
+5 \text { units } \\
\text { none }\end{array}$ \\
\hline
\end{tabular}




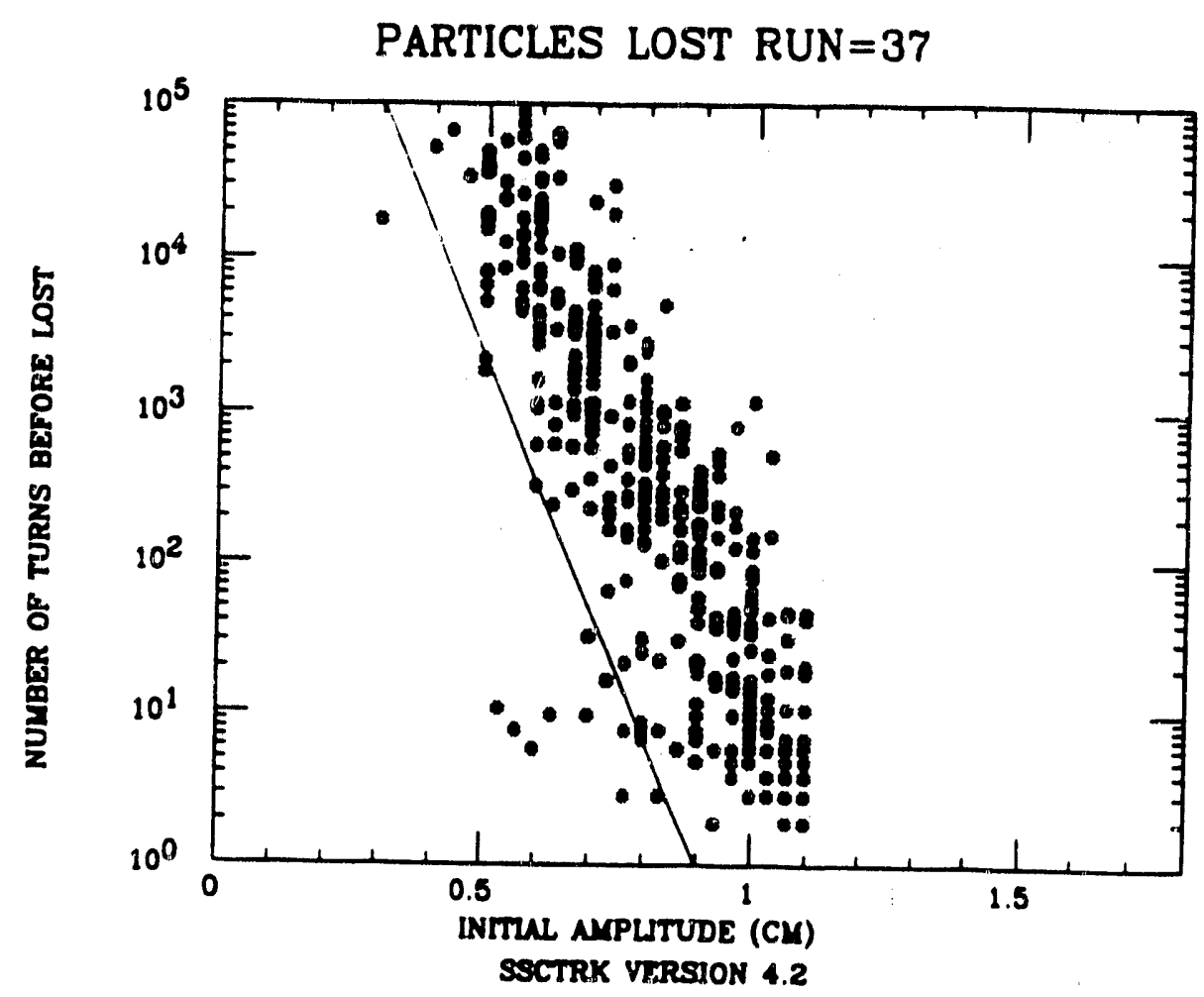

Figure 5. Numerical simulation of the collider ring with $4 \mathrm{~cm}$ dipole errors. This simulation is of the plausibly corrected ring. The line is drawn for comparison with Figure 7 , the $5 \mathrm{~cm}$ dipole error plausibly corrected ring.

PARTICLES LOST RUN $=25$

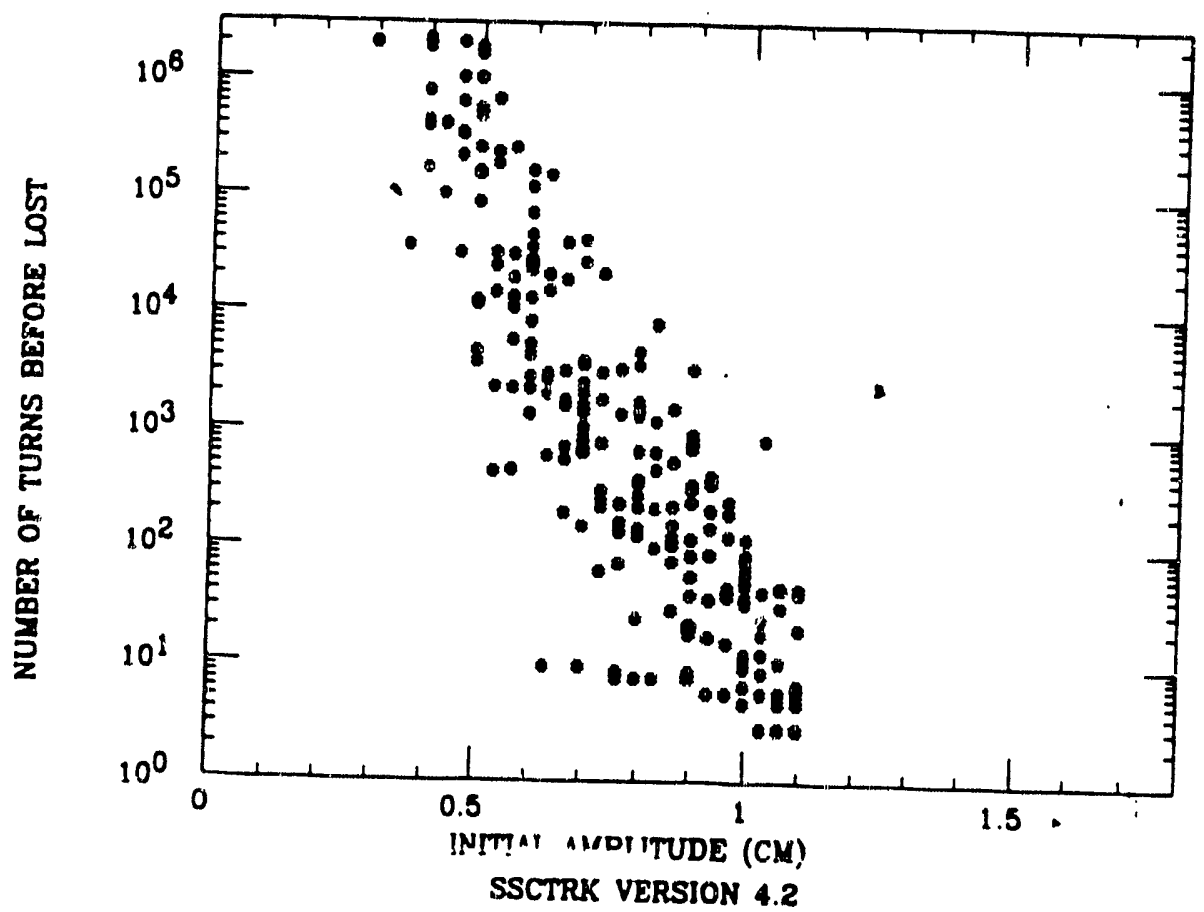

Figure 6. Numerical sirnulation of the collider ring with $4 \mathrm{~cm}$ dipole errors. This simulation is of the plausibly corrected ring. This simulation differs from that of Figure 5 in that only eight lifferent rings are vimulated. This simulation has been extended out to 3 million revolusions. 


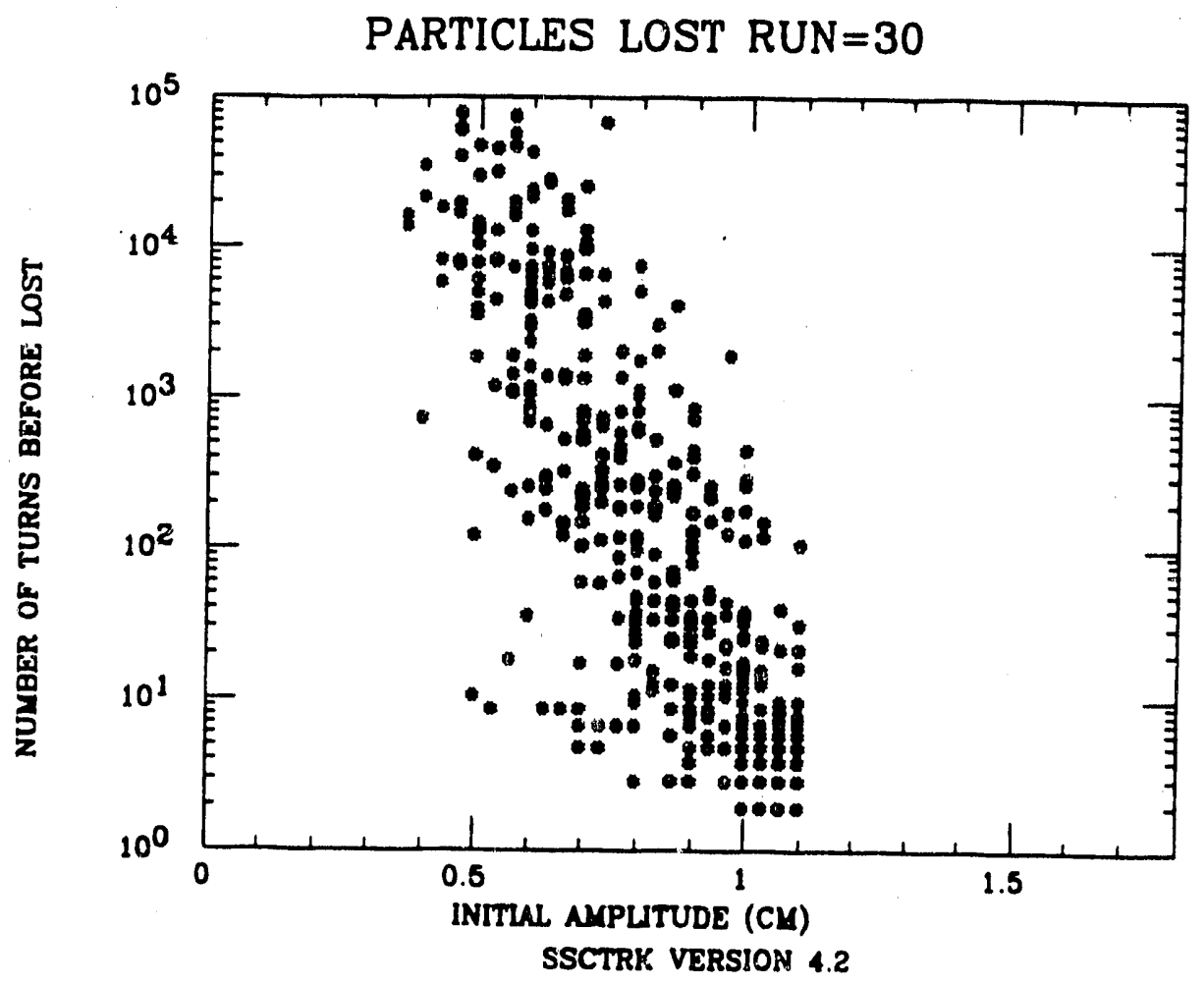

Figure 7. Numerica! simulation of the collider ring with $4 \mathrm{~cm}$ dipole errors. This simulation is of the plausibly corrected ring. In this simulation the value of random sextupole has been increased by a factor of 5 to 2.01 units. It should be compared with Figure 5 where the value of random sextupole was (). 4 units.

When the aperture of the dipole is increased from 4 to $5 \mathrm{~cm}$ both the random and the systematic multipoles decrease. It has been assumed that the random multipoles decrease in the ratio $(4 / 5)^{n+1 / 2}$ and that the systematic multipoles decrease in the ratio $(4 / 5)^{n}$. Figure 8 shows the results of simulating a plausibly tuned machine made with $5 \mathrm{~cm}$ aperture dipoles. The fractional increase in stable betatron amplitude can be estimated by comparing Figure $8(5 \mathrm{~cm})$ with Figure $5(4 \mathrm{~cm})$. The lines in the figures are drawn in an amplitude ratio of 1.6 .

The benefit of increasing the magnet aperture is clearly larger for the plausibly tuned machine than for the highly corrected machine. The ratio 1.6 suggests that the maximum amplitude of stable betatron motion is determined by the value of the product of systematic and random magnet errors. $(1.6=1.28 \times 1.25)$ It is clear that this scaling law cannot be valid for arbitary increases in aperture because the maximum amplitude for stable betatron motion would increase beyond the magnet coils, an absurd notion. The large increase in stable betatron amplitude is a peculiarity of starting with a $4 \mathrm{~cm}$ diameter dipole aperture.

A future report will give simulation results based on a more complete and improved set of magnet errors. There is also clear evidence that splitting the tune values by one unit in order to avoid injection mismatch caused by feed-down systematic skew sextupole would be a desirable improvement. This improvement could be incorporated in the plausibly tuned machine with only a minor increase in the level of correction (a first harmonic skew

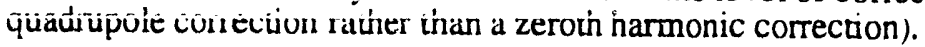


PARTICLES LOST RUN $=36$

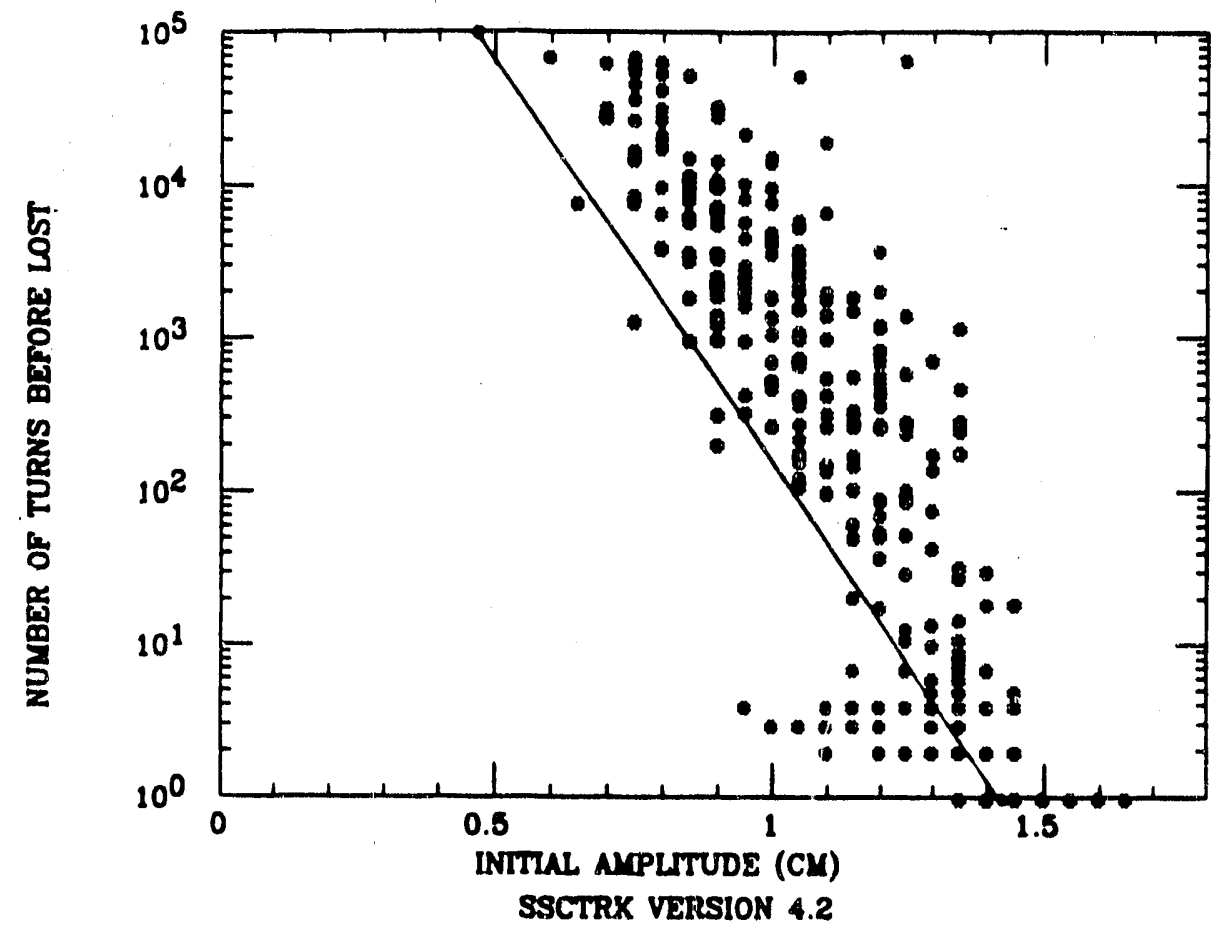

Figure 8. Numerical simulation of the collider ring with $5 \mathrm{~cm}$ dipole errors. This simulation is of the plausibly corrected ring. The line is drawn at an amplitude 1.6 times larger than the line in Figure 5 to illustrate the scaling of stable beiatron aperture.

\section{APPENDIX A}

This appendix contains the job cards used for the SSCTRK simulations discussed in the report.

Run 25: Same errors as run 37. Not shown. 
-END-

DATE FILMED

$127 / 9$ 
